\title{
Serum Lactate/Albumin Ratio as a Predictor of Morbidity and Mortality In Patients with Severe Sepsis and Septic Shock
}

\author{
Essam f. Makram ${ }^{\text {a }}$, Mohamed E. Ibrahim ${ }^{\text {b }}$, Ahmed H. Abdel Rahman ${ }^{\text {a }}$, Karim M. Aboraya ${ }^{a}$
}

a

Department of Anaesthesia

and Intensive Care, Benha Faculty of Medicine, Benha University, b department of nephrology and Internal medicine, Faculty of Medicine Benha University, Egypt

Correspondence to: Karim M. Aboraya, Department of Anaesthesia and Intensive Care, Benha Faculty of Medicine, Benha University, Egypt

Email:

aboraya_karim@yahoo.com

Received: 22 November 2019

Accepted: 17 March 2020

\section{bstract:}

Background: Severe sepsis is common and frequently fatal and represents a significant health care burden. Sepsis is a syndrome of physiologic, pathologic, and biochemical abnormalities induced by infection. Aim of the work: This study examines the ability of serum lactate/albumin ratio to predict outcome regarding organ dysfunction and mortality in severe sepsis and septic shock. Materials and Methods: It is a prospective observational study conducted on 50 patients with severe sepsis and septic shock according to the (SCCM/ESICM/ACCP/ATS/SIS) International Sepsis Definitions Conference 2001 and fifty healthy age matched individuals as a control group. Results: Lactate/albumin ratio was higher on day 0 and 1 in the study group than in control group ( $p$ value $<0.001)$. Also, it was significantly higher in patients that needed mechanical ventilation than patients who didn't ( $p$ value $<0.004$ on day 0 and $<0.001$ on day 1). For patients who needed renal replacement therapy (RRT), lactate/albumin ratio was higher than those who didn't ( $p$ value $<0.001$ for days 0 and 1). When comparing levels of lactate/albumin ratio in survivors and non survivors, we found that it was significantly higher in non survivors ( $p$ value<0.001).Conclusion: Lactate/albumin ratio is a reliable biomarker for prediction of outcome as regards organ dysfunction and mortality in patients with severe sepsis and septic shock.

Key words: Mortality, Septic Shock, Serum Lactate/Albumin Ratio, Severe Sepsis. 


\section{Introduction:}

The incidence and associated mortality and morbidity rates of severe sepsis are commonly underestimated. Sepsis affects more than 750,000 patients each year in the United States; it is the 10th leading cause of death and one of the leading causes for admission to the intensive care unit ${ }^{[1]}$. The estimated mortality from sepsis is $20-30 \%$, meaning that approximately 500,000 patients survive their septic episode annually in the United States alone ${ }^{[2]}$. Mounting evidence has demonstrated that survivors of sepsis have a higher long-term risk of death and a lower health-related quality of life (HRQoL) when compared with the general population ${ }^{[2]}$.

\section{There are different levels of sepsis:}

Systemic inflammatory response syndrome (SIRS) is the presence of two or more of the following:

\begin{tabular}{|l|l|}
\hline Finding & Value \\
\hline Temperature & $<36^{\circ} \mathrm{C}\left(96.8^{\circ} \mathrm{F}\right)$ or $>38^{\circ} \mathrm{C}\left(100.4^{\circ} \mathrm{F}\right)$ \\
\hline Heart rate & $>90 / \mathrm{min}$ \\
\hline $\begin{array}{l}\text { Respiratory } \\
\text { rate }\end{array}$ & $>20 / \mathrm{min}$ or $\mathrm{PaCO} 2<32 \mathrm{mmHg}(4.3 \mathrm{kPa})$ \\
\hline WBC & $\begin{array}{l}<4 \times 10^{9} / \mathrm{L}\left(<4000 / \mathrm{mm}^{3}\right),>12 \times 10^{9} / \mathrm{L} \\
\left(>12,000 / \mathrm{mm}^{3}\right)\end{array}$ \\
\hline
\end{tabular}

Sepsis is defined as SIRS in response to an infectious process ${ }^{[3]}$.

Severe sepsis is defined as sepsis with sepsisinduced organ dysfunction or tissue hypo- perfusion (manifesting as hypotension, elevated lactate, or decreased urine output). Septic shock is severe sepsis plus persistently low blood pressure despite the administration of intravenous fluids ${ }^{[4]}$.

Lactic acid is the normal endpoint of the anaerobic breakdown of glucose in the tissues. The normal blood lactate concentration in unstressed patients is $0.5-1$ mmol/L. Patients with critical illness can be considered to have normal lactate concentrations of less than $2 \mathrm{mmol} / \mathrm{L}$. Hyperlactatemia is defined as a persistent, mild to moderate $(2-4 \mathrm{mmol} / \mathrm{L})$ increase in blood lactate concentration without metabolic acidosis. Serum lactate is a potentially useful biomarker that is widely investigated in patients with critical illness and gave good prognostic values ${ }^{[4]}$.

Albumin is a plasma protein synthesized in the liver. It is the most abundant protein in plasma and constitutes about two-thirds of total protein content. Because it is the main protein in human blood, decreases in albumin due to decreased synthesis or losses result in impaired regulation of intravascular oncotic pressure and manifests as edema. As such, it transports certain hormones (eg, thyroid, estrogen, cortisol). Serum albumin appears to be a reliable prognostic indicator in various contexts. A recent review suggests that serum albumin could be an independent predictor of 
mortality in a wide range of clinical and research settings ${ }^{[5]}$.

Combining both serum lactate and albumin in the form of lactate albumin ratio could yield more accurate prediction about prognosis of critically ill patients specially in septic patients ${ }^{[6]}$.

The aim of this study was to evaluate the ability of lactate/albumin ratio to predict outcome regarding morbidity, mortality, length of ICU stays and extent of organ dysfunctions in patients with severe sepsis and septic shock.

\section{Materials and Methods}

Ethical consideration: All participants signed a written informed consent after with explaining to them the aim of the study prior to the study. Approval was obtained from ethical committee in Faculty of Medicine, Benha University and the study protocol was approved by scientific committee of Benha University

Study design :This study was done as a prospective observational study, that enrolled 50 adult critically ill patients through their ICU stay at Shebin El-Kom teaching hospital in the period from November 2018 to April 2019 diagnosed to have severe sepsis and septic shock according to the SCCM/ESICM/ACCP/ATS/SIS International Sepsis Definitions Conference (Tables 1 and
2) ${ }^{[7]}$ and fifty healthy age matched (without sepsis) adult individuals as a control group.

All patients with the criteria of severe sepsis and septic shock according to the SCCM/ESICM/ACCP/ATS/SIS International Sepsis Definitions Conference were included in this study. They were also treated according to Surviving Sepsis Campaign (International Guidelines for Management of Severe Sepsis and Septic Shock, 2012) ${ }^{[4]}$.

\section{Exclusion criteria included the following:}

Patients with either hepatic dysfunction, renal failure, having any disease in which albumin should be supplemented as liver cirrhosis with ascites, nephrotic syndrome and burns.

All patients underwent the following:

History taking: Including age, gender, weight, co-morbidities (DM, HTN, CVS, HF, COPD, renal failure, liver cell failure, and malignancy).

Thorough Clinical examination: all patients enrolled in this study were evaluated for vital signs (temperature, heart rate, respiratory rate and MAP), Glasgow coma scale (GCS).

Laboratory investigations: Peripheral venous blood specimens were collected on admission for measuring of: Complete blood count (CBC) and differential leucocytic count. Serum lactate in day 0 and $1(\mathrm{mg} / \mathrm{dl})$ using enzymatic colorimetric method by (ROBONIK AN ISO 9001:2002 company ISO 13485: 2003. product: Automatic 
Biochemistry Analyser. Model: Prietest ECO. Serial No: AC0090608RBK).

\section{Sample taking:}

Adequate venous blood samples were withdrawn and centrifuged immediately for serum specimen that is stored frozen at a temperature $-20{ }^{\circ} \mathrm{C}$ under complete aseptic technique.

Normal values of serum lactate: for venous samples: (4.5-19.8 mg/dl)

Commercially available kits of Serum albumin (gram/dl) was used on days 0 and 1 and manufacturing instructions was followed accordingly.

Normal serum albumin range: ( $3.5-5.5$ $\operatorname{gram} / \mathrm{dl})$

Serum albumin and serum lactate levels were collected on admission (day 0) and after 24 hours (day 1) from patients with severe sepsis and septic shock followed by determination of lactate albumin ratio. Serum lactate, albumin and lactate/albumin ratio were correlated to extent of MODS, length of hospital stay and mortality. Serum levels of both lactate and albumin were taken from fifty healthy age matched volunteer individuals as control group. Blood urea $(\mathrm{mg} / \mathrm{dl})$, creatinine $(\mathrm{mg} / \mathrm{dl})$, aspartate transaminase (AST) (U/L), alanine transaminase (ALT) (U/L), prothrombin time (PT) (seconds), international normalized ratio (INR), activated partial thromboplastin time (aPTT) (seconds), bilirubin total and direct $(\mathrm{mg} / \mathrm{dl})$, random blood sugar $(\mathrm{RBS})(\mathrm{mg} / \mathrm{dl})$, $\mathrm{Na}(\mathrm{mEq} / \mathrm{l})$, and $\mathrm{K}(\mathrm{mEq} / \mathrm{l})$, and arterial blood gas (ABG).

Pan-cultures (urine, blood, sputum, and wound) were taken from the study subjects searching for source of sepsis.

Central venous blood samples from the central venous catheter were collected on admission for measuring of central venous oxyhaemoglobin saturation $(\mathrm{ScvO} 2)$.

\section{Statistical analysis:}

Data were coded and entered using the statistical package SPSS (Statistical Package for the Social Science) version 20. Data was summarized using mean, standard deviation, median, minimum and maximum in quantitative data and using frequency (count) and relative frequency (percentage) for categorical data. Comparisons between quantitative variables were done using the non-parametric Mann-Whitney test [8]. Correlations between quantitative variables were done using Spearman correlation coefficient ${ }^{[9]}, p$-values less than 0.05 were considered as statistically significant.

\section{Results}

In this study, we found that serum levels of lactate and lactate/albumin ratio were higher in the study group on days 0 and 1 than control group with statistically significant difference ( $p$ value $<0.001)$ as shown in table 1. 
Table (1): Showing level of serum lactate/albumin ratio in control group and study group

\begin{tabular}{lccc}
\hline $\begin{array}{l}\text { Lactate/ } \\
\text { Albumin } \\
\text { ratio }\end{array}$ & Day 0 & Day 1 & Control group \\
\hline \multirow{2}{*}{ Mean \pm SD } & $26.04 \pm$ & $28.56 \pm$ & \\
& 19.24 & 25.14 & $2.12 \pm 0.72$ \\
p-value & Day 0 \& & Day 0 \& & Day 1 \& \\
& Day 1 & Control & Control \\
& 0.492 & $0.001 *$ & $0.001 *$ \\
\hline
\end{tabular}

p- value $<0.05$ is considered statistically significant . p- value $>0.05$ is considered statistically insignificant .

(*) means statistically significant $\mathrm{p}$ - value.

Lactate/albumin ratio on days 0 and 1 were higher in patients who needed mechanical ventilation and RRT than those who didn't with a statistically significant difference ( $p$ value $<0.001$ ) as shown in tables 2 and 3 respectively.

Table (2): Comparing between patients needed mechanical ventilation with patients didn't need regarding lactate/albumin ratio

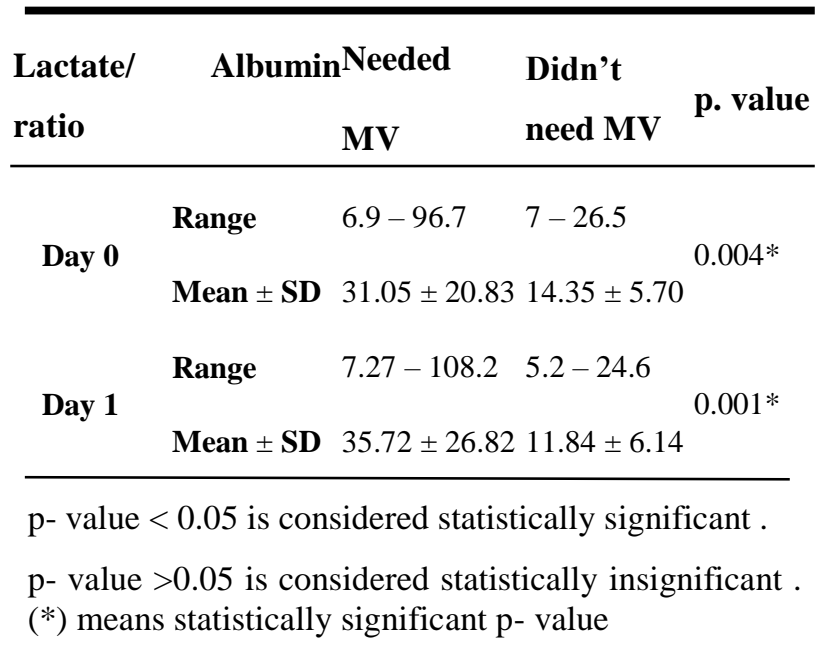

Table (3): Comparing between patients needed renal replacement therapy with patients didn't need regarding lactate/albumin ratio

\begin{tabular}{llll}
\hline $\begin{array}{l}\text { Lactate / Albumin } \\
\text { ratio }\end{array}$ & Needed RRT & \multicolumn{2}{c}{ Didn't need } \\
RRT & p. value \\
\hline \multicolumn{2}{c}{ Range } & $11.15-96.7$ & $6.9-41.9$ \\
Day 0 & & \multicolumn{2}{c}{$\mathbf{0 . 0 0 1 *}$} \\
& Mean \pm SD & $49.73 \pm 26.07$ & $19.34 \pm 9.45$ \\
Day 1 & Range & $8.96-108.2$ & $5.2-72.35$ \\
& & & \multicolumn{2}{c}{$\mathbf{0 . 0 0 1 *}$} \\
& Mean \pm SD & $59.92 \pm 27.91$ & $19.71 \pm 15.65$
\end{tabular}

p- value $<0.05$ is considered statistically significant . p- value $>0.05$ is considered statistically insignificant . (*) means statistically significant p- value.

Lactate and lactate/albumin ratio on days 0 and 1 were higher in non survivor group than survivors with a statistically significant difference ( $p$ value<0.001) as shown in table 4.

Table (4): Showing comparison between levels of serum lactate/albumin ratio in day 0 and day 1 in survivors and non-survivors

\begin{tabular}{|c|c|c|c|c|}
\hline & & Survivors & $\begin{array}{c}\text { Non- } \\
\text { survivors }\end{array}$ & P. value \\
\hline $\begin{array}{l}\text { Ratio } \\
\text { day } 0\end{array}$ & $\begin{array}{l}\text { Range } \\
\text { Mean } \pm \text { SD }\end{array}$ & $\begin{array}{l}7-30.3 \\
17.01 \pm 6.77\end{array}$ & $\begin{array}{l}6.9-96.7 \\
42.09 \pm 23.60\end{array}$ & $0.001^{*}$ \\
\hline $\begin{array}{l}\text { Ratio } \\
\text { day } 1\end{array}$ & Mean \pm SD & $13.87 \pm 6.64$ & $54.67 \pm 24.76$ & $0.001 *$ \\
\hline *) mea & $\begin{array}{l}05 \text { is cons } \\
.05 \text { is cons } \\
\text { tatistically }\end{array}$ & $\begin{array}{l}\text { ered statisti } \\
\text { gnificant } \mathrm{p} \text { - }\end{array}$ & $\begin{array}{l}\text { lly insignific } \\
\text { lue }\end{array}$ & \\
\hline
\end{tabular}

Serum lactate, albumin and lactate/albumin ratio were measured on days 0 and 1 and were 
correlated with extent of organ dysfunction. It was found that there was strong positive correlation ( $\mathrm{r}$ value -0.386 , ) between lactate and lactate/albumin ratio with extent of organ dysfunction with a statistically significant difference ( $p$ value <0.001) and a negative correlation ( $\mathrm{r}$ value - 0.502) between serum albumin on days 0 and 1 and extent of organ dysfunction respectively with statistically significant difference ( $p$ value $<0.001$ ).

On correlating serum lactate, albumin and lactate/albumin ratio with ICU length of stay and we found that there was a statistically insignificant negative correlation between serum lactate and lactate/albumin ratio on days 0 and 1 with $p$ value $(0.087$ and 0.145$)$ respectively and there was a statistically insignificant positive correlation between serum albumin on days 0 and 1 with ( $\mathrm{p}$ values 0.898 and 0.904$)$ respectively.

Also, Lactate/albumin ratio in patients who needed vasopressors for resuscitation was higher than those who didn't need hemodynamic support with a statistically insignificant difference on day 0 and 1 ( $p$ value $=0.151$ and 0.053 respectively).

On the other hand, serum albumin on days 0 and 1 were lower in non survivors than survivors with a statistically significant $(\mathrm{p}$ values $=0.011$ and 0.001 respectively).

\section{Discussion}

Serum lactate is a potentially useful biomarker that is widely investigated in patients with critical illness and gave good prognostic values. It can predict mortality and organ dysfunction ${ }^{[4]}$.

Serum albumin appears to be a reliable prognostic indicator in various contexts. One review suggests that serum albumin could be an independent predictor of mortality in a wide range of clinical and research settings ${ }^{[5]}$. In this study, we found that serum levels of lactate and lactate/albumin ratio were higher in the study group on days 0 and 1 than control group with statistically significant difference ( $p$ value <0.001) whereas serum albumin was significantly lower in the study group on days 0 and 1 than the control group with ( $p$ value $<0.001)$ this was in agreement with Mikkelsen et al.,2009 ${ }^{[10]}$ who stated that serum lactate is higher in septic patients than normal individuals. Also, Wang et al.,2015 ${ }^{[6]}$ found serum lactate/albumin ratio was higher in septic patients. In addition FHY et al., $2002^{[11]}$ found that serum albumin was lower in critically ill patients than normal individuals.

Serum lactate was measured on day 0 and day 1 and was correlated with extent of organ dysfunction, and we found that lactate on day 0 and 1 was positively correlated with extent of organ dysfunction with a statistically significant 
difference ( $p$ value <0.001), which was concordant with Wang et al .2015 ${ }^{[6]}$ who stated that the level of serum lactate was higher in patients with MODS than patients without MODS with statistically significant difference on days 0 and $1 \quad(p$ value $=0.006,0.009$ respectively).

In our study, serum lactate was measured in survivors and non survivors and we found that it was higher in non-survivor group with a statistically significant difference ( $p$ value $<0.001)$. This was in agreement with Jat et al.,2011 ${ }^{[12]}$ who reported that blood lactate was significantly higher in patients with sepsis who died than those who survived. Also, Rabello Filho et al.,2016 ${ }^{[13]}$ found that the higher the blood lactate level in septic patients the higher probability of mortality and in addition, Haas et al.,2016 ${ }^{[14]}$ found that severe hyperlactatemia combined with septicemia is associated with extremely high ICU mortality.

We correlated serum lactate level with ICU length of stay and found that there was a statistically insignificant correlation on days 0 $(p=0.087)$ and $1 \quad(p=0.081)$ which was in agreement with Soliman et al.,2010 ${ }^{[15]}$ who found that a higher serum lactate level is associated with longer ICU length of stay, however this was statistically insignificant ( $p$ value $>0.05)$.

Serum albumin on days 0 and 1 was correlated with extent of organ dysfunction and there was a negative correlation with statistically significant difference $(p<0.001$ which agrees with Spiegel et al.,1994 ${ }^{[16]}$ who mentioned that low serum albumin serves as a marker of morbidity which is an accepted fact that albumin is a negative acute phase reactant that decreases with severe inflammatory states.

Serum albumin was also correlated with length of ICU stay and there was a statistically insignificant difference $(p>0.05)$ between its level and LOS ( length of stay in ICU) which disagreed with Yap et al.,2002 [11] who found significant correlation $(p<0.005)$ between level of admission albumin and LOS in ICU.

Serum albumin was lower in non survivors than survivors with a statistically significant difference on days 0 and $1(p=0.011$ and 0.001 respectively) which matches with Yap et al.,2002 ${ }^{[11]}$ who found that level of serum albumin (on admission) was significantly higher $(p<0.005)$ in survivors than mortality group of critically ill patients.

Lactate/albumin ratio measured on days 0 and 1 showed significant positive correlation ( $p$ value <0.001) with extent of organ dysfunction; the higher the ratio, the more the number of organs affected. This was concordant with Wang et al.,2015 ${ }^{[6]}$ who stated that lactate/albumin ratio is significantly correlated $\quad(p<0.05)$ with subsequent multiorgan failure on day 0 and 1. Lactate/albumin ratio was higher in nonsurvivor group than survivors with a 
statistically significant difference $(p<0.001)$ in both day 0 and 1, which agreed with Wang et al.,2015 ${ }^{[6]}$ who exhibited that lactate/albumin ratio increased in non-survivors with statistically significant difference $(p=0.012)$.

Upon correlating lactate/albumin ratio on days 0 and 1 with ICU length of stay, there was a negative correlation ( $\mathrm{r}$ value - 0.166 , 0.209) with a statistically insignificant difference $(p>0.05)$ on day 0 and 1 .

Lactate/albumin ratio was higher in patients who needed mechanical ventilation than those who didn't with a statistically significant difference $(p<0.001)$ which disagree with Wang et al.,2015 ${ }^{[6]}$ who found statistically insignificant difference $(p=0.5839)$ between patients who needed MV and lactate/albumin ratio. Lactate/albumin ratio in patients who needed vasopressors for resuscitation was insignificantly higher $(p>0.05)$ than those who didn't need hemodynamic support on day 0 and day 1 , which is concordant with Wang et al.,2015 ${ }^{[6]}$ who found a statistically insignificant difference $(p=0.8636)$. between patients who needed hemodynamic support and those who didn't. Lactate/albumin ratio was significantly higher $(p<0.001)$ in patients who needed renal replacement therapy than in those who didn't need it on day 0 and 1 .

\section{Conclusion:}

This study concluded that Lactate/albumin ratio is a promising and readily available biomarker in prognosticating severe sepsis and septic shock.

Lactate/albumin ratio is thought to be an applicable biomarker for prediction of outcome, as regards organ dysfunction and mortality in patients with severe sepsis and septic shock.

\section{References}

1. Longo CJ, Heyland DK, Fisher HN, Fowler RA, Martin CM, Day AG. (2007): A long-term followup study investigating health-related quality of life and resource use in survivors of severe sepsis: comparison of recombinant human activated protein C with standard care. Critical Care. 11(6):R128.

2. Erickson SE, Martin GS, (2008): Effect of sepsis therapies on health-related quality of life. Crit Care; 12(1):109.

3. Soong J, Soni. (2012): Sepsis: Recognition and treatment. Clinical Medicine. 276-80.

4. Dellinger RP, Levy MM, Rhodes A, Annane D, Gerlach H, Opal SM, et al. (2013): Surviving Sepsis Campaign: international guidelines for management of severe sepsis and septic shock. Intensive care medicine. 39(2):165-228.

5. Dupuy AM, Philippart F, Péan Y, Lasocki S, Charles PE, Chalumeau M, et al. (2013): Role of biomarkers in the management of antibiotic therapy: an expert panel review: I-currently available biomarkers for clinical use in acute infections. Annals of Intensive Care; 3(1):1.

6. Wang B, Chen G, Cao Y, Xue J, Li J, Wu Y, (2015): Correlation of lactate/albumin ratio level to organ failure and mortality in severe sepsis and septic shock. Journal of Critical Care. 30(2):271275.

7. Levy MM, Fink MP, Marshall JC, Abraham E, Angus D, Cook D, et al. (2003): 2001 $\mathrm{sccm} / \mathrm{esicm} / \mathrm{accp} / \mathrm{ats} / \mathrm{sis}$ international sepsis 
definitions conference. Intensive care medicine. 29(4):530-8.

8. Chan YH. (2003a): Biostatistics102: Quantitative Data - Parametric \& Non-parametric Tests. Singapore Med J. 44(8):391-396.

9. Chan YH. (2003b): Biostatistics 104: Correlational Analysis. Singapore Med J. 44(12): 614-619

10. Mikkelsen ME, Miltiades AN, Gaieski DF, Goyal M, Fuchs BD, Shah CV, et al. (2009): Serum lactate is associated with mortality in severe sepsis independent of organ failure and shock. Critical care medicine. 37(5):1670-7.

11. Yap FH, Joynt GM, Buckley TA, Wong EL. (2002): Association of serum albumin concentration and mortality risk in critically ill patients. Anaesthesia and intensive care. 30(2):202-7.
12. Jat KR, Jhamb U, Gupta VK, (2011): Serum lactate levels as the predictor of outcome in pediatric septic shock. Indian Journal of Critical Care Medicine; 15(2):102.

13. Rabello Filho R, Rocha LL, Corrêa TD, Pessoa CM, Colombo G, Assuncao MS. (2016): Blood lactate levels cutoff and mortality prediction in sepsis - time for a reappraisal? A retrospective cohort study. Shock (Augusta, Ga.). 46(5):480.

14. Haas SA, Lange T, Saugel B, Petzoldt M, Fuhrmann V, Metschke M, et al. (2016): Severe hyperlactatemia, lactate clearance and mortality in unselected critically ill patients. Intensive care medicine. 42(2):202-10.

15. Soliman HM, Vincent JL. (2010): Prognostic value of admission serum lactate concentrations in intensive care unit patients. Acta Clin Belg. 65(3):176-81.

16. Spiegel DM, Breyer JA. (1994): Serum albumin: a predictor of long term outcome in peritoneal dialysis patients. Am J Kidney Dis; 23(2):283-5.

To cite this article: Essam f. Makram , Mohamed E. Ibrahim, Ahmed H. Abdel Rahman, Karim M. Aboraya. Serum lactate/albumin ratio as a predictor of morbidity and mortality in patients with severe sepsis and septic shock. BMFJ. 2020; 37(1): 220-228. DOI:10.21608/bmfj.2020.19931.1171 